\title{
Improved convergence in block copolymer self-consistent field theory by Anderson mixing
}

\author{
R. B. Thompson, ${ }^{\text {a) }}$ K. $\varnothing$. Rasmussen, and T. Lookman \\ Theoretical Division, Los Alamos National Laboratory, Los Alamos, New Mexico 87545
}

(Received 17 September 2003; accepted 3 October 2003)

\begin{abstract}
A modification to real space polymeric self-consistent field theory algorithms that greatly improves the convergence properties is presented. The method is based on Anderson mixing [D. G. Anderson, J. Assoc. Comput. Mach. 12, 547 (1965)], and each iteration computed takes negligibly longer to perform than with other methods, but the number of iterations required to reach a high accuracy solution is greatly reduced. No a priori knowledge of possible phases is required to apply this method. We apply our approach to a standard diblock copolymer melt, and demonstrate iteration reductions of more than a factor of 5 in some cases. (C) 2004 American Institute of Physics.
\end{abstract}

[DOI: $10.1063 / 1.1629673$ ]

\section{INTRODUCTION}

Self-consistent field theory (SCFT) has proven itself to be one of the best theoretical methods for studying block copolymer systems, ${ }^{1}$ and can also be applied to general polymeric fluids. ${ }^{2}$ It is a mean-field theory, which yields morphologies, free energies, entropies, enthalpies, ${ }^{1}$ bridging fractions, ${ }^{3-5}$ and also some macroscopic mechanical properties. ${ }^{6-8}$ Comparison with experiments have shown it to be quantitatively as well as qualitatively reliable, when solved to high accuracy. ${ }^{1}$ Originally, SCFT was solved in real space, ${ }^{9-11}$ which limited its precision. A powerful spectral approach ${ }^{12}$ has since emerged that allows dependable phase diagrams to be produced. More recently, there has been a turn back towards a real space method of solution because of the ease of implementation and the ability to predict morphologies. ${ }^{4,13-16}$ This approach has been given a boost by a pseudospectral method of solution, ${ }^{15,16}$ which greatly increases the efficiency of the real space method. Many such modern real space methods depend upon a "simple mixing" iterative technique for solving the selfconsistent set of equations. ${ }^{4,13-16}$ This approach can require a large number of iterations if the free energy is determined to a reasonable level of accuracy. In this article, we suggest an alternative method that converges much faster, and can speed up real space SCFT calculations in some cases by more than a factor of 5 .

The method we suggest was originally presented by Anderson ${ }^{17}$ in a different context, and is therefore sometimes known as "Anderson mixing." It has been clearly outlined by $\mathrm{Ng},{ }^{18}$ and was introduced in the context of SCFT by Schmid ${ }^{19,20}$ in order to study lipid monolayers. Schmid used a variant of Anderson mixing, which we find does not speed up the convergence of polymeric SCFT systems with respect to simple mixing. We present an implementation that combines the rapid convergence of standard Anderson mixing with the "combinatorial screening" feature ${ }^{13}$ of the pseu-

\footnotetext{
a) Author to whom correspondence should be addressed. Electronic mail: rthompson@lanl.gov
}

dospectral real space algorithm for block copolymer systems.

The neat diblock copolymer system has been extensively studied with SCFT, ${ }^{1}$ and so it makes an appropriate "fruitfly" for us to demonstrate our algorithm. It should be noted however that the method is applicable to all block copolymer, polymer blend, and polymeric fluids to which one can apply SCFT. $^{2}$

\section{THEORY}

SCFT has been described extensively elsewhere., 1,2,20 Here we will assume a basic familiarity with the theory and write the free energy for an AB diblock copolymer melt as

$$
\begin{aligned}
\frac{F}{n k_{B} T}= & -\ln Q+\frac{1}{V} \int d \mathbf{r}\left[\chi N \varphi_{\mathrm{A}}(\mathbf{r}) \varphi_{\mathrm{B}}(\mathbf{r})-w_{\mathrm{A}}(\mathbf{r}) \varphi_{\mathrm{A}}(\mathbf{r})\right. \\
& \left.-w_{\mathrm{B}}(\mathbf{r}) \varphi_{\mathrm{B}}(\mathbf{r})\right] .
\end{aligned}
$$

In Eq. (1), $\varphi_{\mathrm{A}}(\mathbf{r})$ and $\varphi_{\mathrm{B}}(\mathbf{r})$ are the local monomer ${ }^{21}$ densities of the $\mathrm{A}$ and $\mathrm{B}$ chemical species, respectively. Conjugate to these densities are the chemical potential fields $w_{\mathrm{A}}(\mathbf{r})$ and $w_{\mathrm{B}}(\mathbf{r}) . \quad \chi$ is the Flory-Huggins interaction parameter between $\mathrm{A}$ and $\mathrm{B}$ monomers, based on a monomer volume of $\rho_{0}^{-1}$ and a degree of polymerization $N$. Further, $V$ is the volume of the system, $n$ is the number of molecules in the volume, $k_{B}$ is Boltzmann's constant, and $T$ is the temperature. The partition function $Q$ of a single diblock molecule subject to the fields $w_{\mathrm{A}}(\mathbf{r})$ and $w_{\mathrm{B}}(\mathbf{r})$ is given by

$$
Q=\int d \mathbf{r} q(\mathbf{r}, s) q^{\dagger}(\mathbf{r}, s),
$$

where the propagators $q(\mathbf{r}, s)$ and $q^{\dagger}(\mathbf{r}, s)$ are solutions to modified diffusion equations given elsewhere. ${ }^{1}$ The $s$ parameter in the propagators follows the contour of the diblock from one end $(s=0)$ through the junction point $(s=f)$ and to the other end $(s=1)$. The free energy (1) can be determined by solving the self-consistent set of equations

$$
\begin{aligned}
& w_{\mathrm{A}}(\mathbf{r})=\chi N \varphi_{\mathrm{B}}(\mathbf{r})+\xi(\mathbf{r}), \\
& w_{\mathrm{B}}(\mathbf{r})=\chi N \varphi_{\mathrm{A}}(\mathbf{r})+\xi(\mathbf{r}),
\end{aligned}
$$


TABLE I. Iterations required for a given accuracy tolerance. The first row gives the number of iterations required to reach a relative accuracy of $10^{-3}$ in the fields using simple mixing only. Subsequent rows give the additional iterations required to reach the indicated accuracy. The last row gives the total number of iterations required to reach an accuracy of $10^{-16}$. The results given here are for the lamellar system shown in Fig. 1.

\begin{tabular}{lccccc}
\hline \hline & \multicolumn{2}{c}{ Simple mixing } & & \multicolumn{2}{c}{ Anderson mixing } \\
\cline { 2 - 3 } \cline { 5 - 6 } Tolerance & Iterations & Free energy & & Iterations & Free energy \\
\hline $10^{-3}$ & 100 & 4.385597865 & & $\cdots$ & $\ldots$ \\
$10^{-4}$ & 36 & 4.393744754 & & 4 & 4.388714217 \\
$10^{-5}$ & 59 & 4.392150644 & & 3 & 4.392041233 \\
$10^{-6}$ & 36 & 4.391962299 & & 3 & 4.392174754 \\
$10^{-7}$ & 59 & 4.391969470 & & 4 & 4.391997179 \\
$10^{-8}$ & 60 & 4.391973279 & & 4 & 4.392009709 \\
$10^{-9}$ & 59 & 4.391974733 & & 3 & 4.391961370 \\
$10^{-10}$ & 36 & 4.391975785 & & 5 & 4.391979954 \\
$10^{-11}$ & 60 & 4.391975693 & & 3 & 4.391974741 \\
$10^{-12}$ & 59 & 4.391975657 & & 4 & 4.391975490 \\
$10^{-13}$ & 60 & 4.391975638 & & 3 & 4.391975489 \\
$10^{-14}$ & 36 & 4.391975628 & & 6 & 4.391975647 \\
$10^{-15}$ & 59 & 4.391975629 & & 4 & 4.391975630 \\
$10^{-16}$ & 60 & 4.391975629 & & 8 & 4.391975622 \\
Totals & $\mathbf{7 7 9}$ & & & $\mathbf{1 5 4}$ & \\
\hline \hline
\end{tabular}

$$
\begin{aligned}
& \varphi_{\mathrm{A}}(\mathbf{r})+\varphi_{\mathrm{B}}(\mathbf{r})=1, \\
& \varphi_{\mathrm{A}}(\mathbf{r})=\frac{V}{Q} \int_{0}^{f} d s q(\mathbf{r}, s) q^{\dagger}(\mathbf{r}, s), \\
& \varphi_{\mathrm{B}}(\mathbf{r})=\frac{V}{Q} \int_{f}^{1} d s q(\mathbf{r}, s) q^{\dagger}(\mathbf{r}, s),
\end{aligned}
$$

where $\xi(\mathbf{r})$ is a field that enforces incompressibility.

Typical real space approaches to solving Eqs. (3)-(7) consist of generating random fields $w_{\mathrm{A}}(\mathbf{r})$ and $w_{\mathrm{B}}(\mathbf{r})$ and solving for the densities $\varphi_{\mathrm{A}}(\mathbf{r})$ and $\varphi_{\mathrm{B}}(\mathbf{r})$, then recalculating the fields. Details on the specifics of such iterative algorithms can be found in Refs. 2, 4, and 13-16. Iteration through direct substitution of the fields is unstable, so a "simple mixing" update is usually performed according to $^{2,4,13,14}$

$$
w_{i+1}^{\text {in }}=(1-\lambda) w_{i}^{\text {in }}(\mathbf{r})+\lambda w_{i}^{\text {out }}(\mathbf{r}),
$$

where $w_{i}^{\text {in }}(\mathbf{r})$ is the input value for iteration $i, w_{i}^{\text {out }}(\mathbf{r})$ is the resultant field, and $w_{i+1}^{\text {in }}$ is the field to be input for the next iteration. $\lambda$ is a parameter chosen sufficiently small to ensure stability, typically $\lesssim 0.1$. The prescription (8) is usable, but slow and sometimes unstable. Some authors ${ }^{15,16}$ have modified Eq. (8) slightly using density information in an attempt to increase the stability.

In this article, we use a combination of Eq. (8) and a method described by $\mathrm{Ng}^{18}$ and Schmid. ${ }^{19}$ We define a deviation function as

$$
d_{i}(\mathbf{r})=w_{i}^{\text {out }}(\mathbf{r})-w_{i}^{\text {in }}(\mathbf{r}),
$$

from which we can specify a total deviation through the inner product

$$
(g(\mathbf{r}), h(\mathbf{r})) \equiv \int d \mathbf{r} g(\mathbf{r}) h(\mathbf{r}),
$$

where $g(\mathbf{r})$ and $h(\mathbf{r})$ are arbitrary functions. Our total deviation is then

$$
d_{i}^{\mathrm{tot}}=\left|\frac{\left(d_{i}(\mathbf{r}), d_{i}(\mathbf{r})\right)}{\left(w_{i}^{\text {out }}(\mathbf{r}), w_{i}^{\text {out }}(\mathbf{r})\right)}\right|,
$$

which follows the definition of Schmid and Müller ${ }^{10}$ rather than that of Ng. ${ }^{18}$ Simple mixing is performed until a certain tolerance is reached where a morphology has begun to develop. Typically, $d^{\text {tot }} \sim 10^{-3}$ is sufficient, although in many cases $d^{\text {tot }} \sim 10^{-2}$ or $10^{-1}$ will do. We then switch to the $\mathrm{Ng}$ procedure, and hence define the matrix

$$
U_{n m}=\left(d_{i}(\mathbf{r})-d_{i-n}(\mathbf{r}), d_{i}(\mathbf{r})-d_{i-m}(\mathbf{r})\right),
$$

and vector

$$
V_{n}=\left(d_{i}(\mathbf{r})-d_{i-n}(\mathbf{r}), d_{i}(\mathbf{r})\right),
$$

with dimensions $n=m$, which are arbitrary. ${ }^{22}$ Calculating the coefficients,

$$
A_{n}=U_{n m}^{-1} V_{n},
$$

allows us to use the prescription

$$
w_{i+1}^{\text {out }}(\mathbf{r})=w_{i}^{\text {out }}+\sum_{n} A_{n}\left(w_{i-n}^{\text {out }}(\mathbf{r})-w_{i}^{\text {out }}(\mathbf{r})\right) .
$$

One can see that whereas the simple mixing prescription (8) mixed the input and output of a single iteration using an arbitrary mixing parameter $\lambda$ to obtain the input for the next iteration, the Anderson mixing prescription (15) mixes the output of several iterations and calculates the best mixing parameters possible assuming a linear system. Obviously, the SCFT system (3)-(7) is not linear, but the Anderson mixing procedure is able to correct for occasional bad steps.

We should point out that Schmid has also used a combination of simple mixing and Anderson mixing in SCFT applied to lipid molecules, ${ }^{19}$ however, that approach differs 


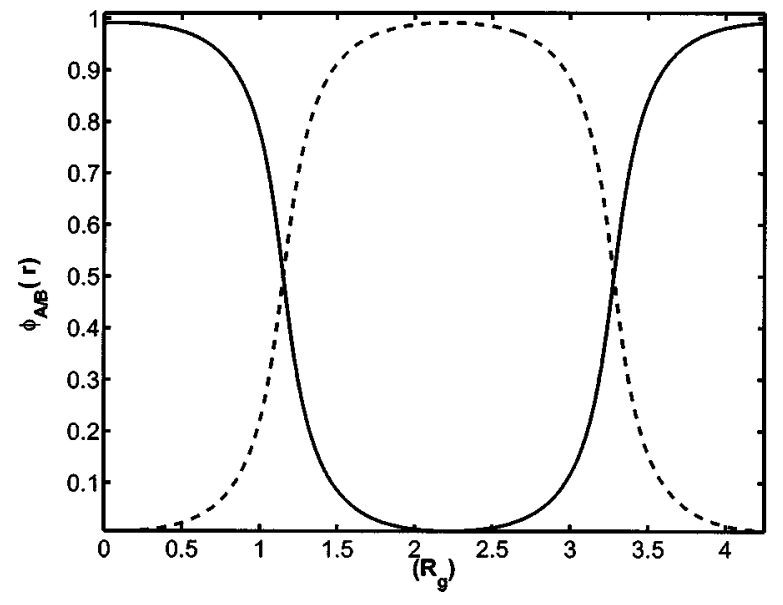

FIG. 1. Lamellar morphology of a diblock copolymer with $f=0.5$ and $\chi N$ $=25$. The solid line is the $\mathrm{A}(\mathrm{B})$ phase and the dashed line is the $\mathrm{B}(\mathrm{A})$ phase.

from our method. Schmid defined formulas analogous to Eq. (15) for both the input and output fields, and these two results were combined through simple mixing. ${ }^{23}$ We find that the Schmid method does not noticeably reduce the iteration count for polymeric systems with respect to pure simple mixing. Instead, we have used simple mixing alone at the start of the algorithm to distill out a morphology, followed by Anderson mixing alone to rapidly converge the solution to a high relative accuracy in the fields. We have found that using Anderson mixing directly from random fields can be unstable, or will result in highly metastable solutions.

\section{RESULTS AND DISCUSSION}

The algorithm described in the previous section has been applied to a diblock copolymer melt; the corresponding SCFT equations were also given in Sec. II. The results for a system with $\chi N=25$ and $f=0.5$ are shown in Table I. The first column shows the relative accuracy achieved in the fields; the second and third columns give the iteration count and free energy, respectively, for a simple mixing calculation. The fourth and fifth columns are the same as the second and third, except that Anderson mixing was used. The first row gives the number of iterations required to reach a relative accuracy of $10^{-3}$ in the fields using simple mixing only. Subsequent rows give the additional iterations required to reach the indicated accuracy. The last row gives the total number of iterations required to reach an accuracy of $10^{-16}$. The final morphology is shown in Fig. 1. Bearing in mind that an Anderson iteration takes negligibly longer to perform than a simple mixing iteration, it is clear that the Anderson method is more than five times faster than the simple mixing method. Since at $\chi N=25$ and $f=0.5$, the diblock copolymer system is known to be in a lamellar state, Table I was generated using a one-dimensional code. Two- and threedimensional runs on other morphologies however, have given similar benefits.

From our experience, the lamellar morphology can take longer to "anneal" when using simple mixing than some other morphologies, such as the body-centered-cubic (bcc) spherical phase. Indeed, we have found the bcc phase particularly amenable to the traditional simple mixing approach. Even for this case, however, a significant benefit is realized by using Anderson mixing, as shown in Table II. One can see from the total iterations that Anderson mixing is more than twice as fast as simple mixing for the bcc morphology. The final morphology produced is shown in Fig. 2.

The benefits displayed in Tables I and II are the result of starting from random fields. If a reasonable first guess is already available for a given morphology, then the benefits of the Anderson approach can be much greater. Work has been done on the elastic properties of block copolymer melts, ${ }^{7,8}$ where deformations about an equilibrium structure are investigated using SCFT. Such calculations, when done in real space, ${ }^{8}$ could be performed far more efficiently and to a much higher accuracy using the current approach.

In Tables I and II, the increased accuracy in the free energy reflects only the increased accuracy of the iterated fields in a given discretization. The number of real space grid points, or the discretization of the timelike contour parameter $s$ will also limit the free energy accuracy. Certainly, the

TABLE II. Same as Table I except for the bcc spherical phase shown in Fig. 2. The last row gives the total number of iterations required to reach an accuracy of $10^{-15}$.

\begin{tabular}{lrrrrr}
\hline \hline & \multicolumn{2}{c}{ Simple mixing } & & \multicolumn{2}{c}{ Anderson mixing } \\
\cline { 2 - 3 } \cline { 5 - 6 } Tolerance & Iterations & Free energy & & Iterations & Free energy \\
\hline $10^{-3}$ & 720 & 3.842855605 & & $\cdots$ & $\ldots$ \\
$10^{-4}$ & 29 & 3.842396475 & & 8 & 3.844175772 \\
$10^{-5}$ & 31 & 3.844500524 & & 3 & 3.845097114 \\
$10^{-6}$ & 31 & 3.845272439 & & 4 & 3.845547576 \\
$10^{-7}$ & 43 & 3.845469768 & & 6 & 3.845473906 \\
$10^{-8}$ & 129 & 3.845444712 & & 15 & 3.845438991 \\
$10^{-9}$ & 144 & 3.845415456 & & 15 & 3.845411627 \\
$10^{-10}$ & 145 & 3.845404670 & & 18 & 3.845403465 \\
$10^{-11}$ & 145 & 3.845400721 & & 15 & 3.845400713 \\
$10^{-12}$ & 145 & 3.845399272 & & 17 & 3.845399256 \\
$10^{-13}$ & 147 & 3.845398735 & & 20 & 3.845398669 \\
$10^{-14}$ & 150 & 3.845398539 & & 16 & 3.845398515 \\
$10^{-15}$ & 151 & 3.845398468 & & 13 & 3.845398466 \\
Totals & $\mathbf{2 0 1 0}$ & & & $\mathbf{8 7 0}$ & \\
\hline \hline
\end{tabular}




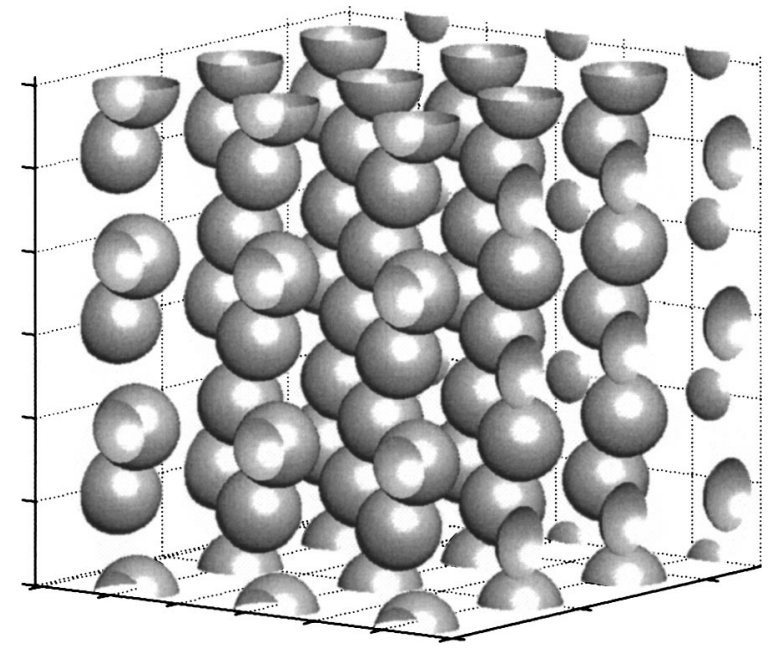

FIG. 2. bcc morphology of a diblock copolymer with $f=0.2$ and $\chi N=25$. The calculated pattern is repeated three times in every direction. The isosurfaces show contours of equal $\mathrm{A}$ and $\mathrm{B}$ densities, that is, contours at 0.5 A(B) density.

present method allows sufficient accuracy at a given discretization to remove one previously important limiting factor. This approach also allows one to more quickly get results when interpolating a known solution to a finer discretization. It may be that in time the real space method free energy will allow the construction of phase diagrams competitive with the more sophisticated basis function method. ${ }^{24}$

\section{CONCLUSIONS}

An algorithm for solving polymeric SCFT equations based on Anderson mixing has been presented, which can greatly speed up convergence. For a lamellar morphology, we find an improvement of more than a factor of 5 . For a bcc spherical morphology, which is among the easiest to solve with simple mixing, we still see a benefit of more than a factor of 2. In more complicated systems or morphologies where simple mixing iterations oscillate significantly before an accurate free energy is found, we expect even greater improvements than those reported here.

The Anderson mixing method can be used by itself for cases where a reasonable initial guess is known. From Tables I and II, it can be seen that Anderson mixing reduces iterations for a good initial guess by a factor of roughly 10 . If starting from random fields, the method can be used subsequent to simple mixing. The higher accuracy of the free energy that is obtainable using this method relates only to the relative accuracy of the iterated fields. To make phase diagram calculations competitive with the spectral method, ${ }^{12}$ other improvements in the real space approach are required.
One possibility is the interpolation to denser meshes. The present method of solution makes such an approach much more feasible.

Lastly, the Anderson mixing method can be applied to field theoretic simulations (FTS), as well. The FTS method involves numerically solving the exact partition function of the polymer fluid model, and does not use the mean-field approximation. This approach has been restricted to two dimensions previously because of the computational intensity. Since good initial guesses are always used for these systems, the application of Anderson mixing could well make threedimensional FTS calculations possible.

\section{ACKNOWLEDGMENTS}

Work at the Los Alamos National Laboratory is performed under the auspices (Contract No. W-7405-ENG-36) of the U.S. Department of Energy. One of the authors (R.B.T.) acknowledges helpful communication with F. Schmid.

${ }^{1}$ M. W. Matsen, J. Phys.: Condens. Matter 14, R21 (2002), and references therein.

${ }^{2}$ G. H. Fredrickson, V. Ganesan, and F. Drolet, Macromolecules 35, 16 (2002), and references therein.

${ }^{3}$ K. Ø. Rasmussen, E. M. Kober, T. Lookman, and A. Saxena, J. Polym. Sci., Part B: Polym. Phys. 41, 104 (2003).

${ }^{4}$ F. Drolet and G. H. Fredrickson, Macromolecules 34, 5317 (2001).

${ }^{5}$ M. W. Matsen and R. B. Thompson, J. Chem. Phys. 111, 7139 (1999).

${ }^{6}$ M. B. Kossuth, D. C. Morse, and F. S. Bates, J. Rheol. 43, 167 (1999).

${ }^{7}$ C. A. Tyler and D. C. Morse, Macromolecules 36, 3764 (2003).

${ }^{8}$ R. B. Thompson, K. Ø. Rasmussen, and T. Lookman (unpublished).

${ }^{9}$ M. D. Whitmore and J. D. Vavasour, Acta Polym. 46, 341 (1995).

${ }^{10}$ F. Schmid and M. Müller, Macromolecules 28, 8639 (1995).

${ }^{11}$ K. M. Hong and J. Noolandi, Macromolecules 14, 727 (1981).

${ }^{12}$ M. W. Matsen and M. Schick, Phys. Rev. Lett. 72, 2660 (1994).

${ }^{13}$ F. Drolet and G. H. Fredrickson, Phys. Rev. Lett. 83, 4317 (1999).

${ }^{14}$ R. B. Thompson, V. V. Ginzburg, M. W. Matsen, and A. C. Balazs, Macromolecules 35, 1060 (2002).

${ }^{15}$ G. Tzeremes, K. Ø. Rasmussen, T. Lookman, and A. Saxena, Phys. Rev. E 65, 041806 (2002).

${ }^{16}$ K. Ø. Rasmussen and G. Kalosakas, J. Polym. Sci., Part B: Polym. Phys. 40, 1777 (2002).

${ }^{17}$ D. G. Anderson, J. Assoc. Comput. Mach. 12, 547 (1965); V. Eyert, J. Comput. Phys. 124, 271 (1996).

${ }^{18}$ K.-C. Ng, J. Chem. Phys. 61, 2680 (1974).

${ }^{19}$ F. Schmid, Phys. Rev. E 55, 5774 (1997).

${ }^{20}$ F. Schmid, J. Phys.: Condens. Matter 10, 8105 (1998).

${ }^{21}$ Strictly speaking, $\varphi_{\mathrm{A}}(\mathbf{r})$ and $\varphi_{\mathrm{B}}(\mathbf{r})$ are local segment densities, each coarse grained segment consisting of a number of chemical monomers.

${ }^{22}$ For more complex situations, a larger $n$ can be taken, although the solution of the $n \times n$ matrix will slow the iteration if $n$ is too large. We find that $n=3$ allows the algorithm to robustly converge without noticeably slowing the iterations.

${ }^{23}$ In this case, the objective was not to increase the speed of convergence, but rather to stabilize the convergence. To this end, the Schmid algorithm was applied to spherical harmonic coefficients of the fields rather than to the fields in real space. In this work, we iterate on the real space fields.

${ }^{24}$ M. W. Matsen and F. Bates, Macromolecules 29, 1091 (1996). 